\title{
L'ÉVALUATION DE LA PERFORMANCE ECONOMIQUE DES ENTREPRISES PUBLIQUES AFRICAINES PAR LA MÉTHODE DES COMPTES DE SURPLUS
}

\author{
par \\ Mapapa MBANGALA* \\ Université de Liège, Belgique
}

\begin{abstract}
RÉSUMÉ**: Cet article utilise la méthode de comptes de surplus pour évaluer la performance économique de sept entreprises ferroviaires africaines. Cette méthode montre le rôle de ces entreprises publiques dans la création et la distribution de richesses. L'analyse met en lumière la contribution de chaque facteur à l'obtention de gains ou de pertes de productivité. En même temps, elle indique les différents partenaires qui bénéficient des avantages créés par les compagnies de chemin de fer.
\end{abstract}

\section{Introduction}

Dans les pays en voie de développement, comme en Afrique noire, il existe un grand nombre d'entreprises publiques, notamment dans les secteurs de l'électricité et des transports et communications (Mishra et Ravishankar 1986). ${ }^{1}$ Indépendamment de la doctrine politique ou de l'idéologie professée par chaque gouvernement, ce secteur public a, au

\footnotetext{
* Nous tenons à remercier les lecteurs anonymes pour la pertinence de leurs remarques et commentaires. Nos remerciements s'adressent surtout à Sergio Perelman pour les conseils et suggestions qu'il nous a formulés.

** Abstract at the end of the article; Zusammenfassung am Ende des Artikels; resúmen al fin del artículo.

1 Il s'agit des entreprises contrôlées par l'Etat et qui sont censées tirer l'essentiel de leur revenu de la vente de biens et de services, qui jouissent de l'autonomie financière et qui ont une identité juridique distincte.
} 
fil des ans, pris des proportions considérables en occupant une place importante dans le contrôle des leviers de commande de l'économie (Nations Unies 1986). ${ }^{2}$

Cependant on assiste en cette fin du $20^{\text {ème }}$ siècle, marquée par la globalisation de l'économie, à une libéralisation ou privatisation systématique des entreprises publiques africaines (Finifter et Trevoux 1992) qui étaient restées la chasse gardée des pouvoirs publics. Les arguments avancés sont que ces entreprises ont un niveau de rentabilité financière et/ou de productivité qui est généralement considéré comme très bas, sinon médiocre (Nellis 1988, Kempe 1999). La Banque Mondiale (1994) présente un tableau d'ensemble catastrophique. Elle considère que ces entreprises sont devenues une menace pour l'Etat à cause de leurs mauvaises performances et de leurs résultats financiers désastreux.

La question fondamentale que nous nous posons est de savoir si toutes les entreprises publiques quel que soit leur champ d'activité doivent être évaluées à l'aune du seul critère de rentabilité financière.

Pour répondre à cette question, cet article utilise la méthode de comptes de surplus pour évaluer la performance économique de sept entreprises ferroviaires africaines. Cette méthode montre le rôle que jouent ces entreprises dans la création et la distribution de richesses. L'analyse met en lumière la contribution de chaque facteur à l'obtention de gain ou de perte de productivité. En même temps, elle indique les différents partenaires de ces entreprises qui bénéficient des avantages que celles-ci créent.

Le travail est divisé en trois parties. La première partie indique les raisons qui portent à penser qu'il peut y avoir une méthodologie spécifique d'évaluation de la performance des entreprises publiques. Le choix des critères de performance doit faire référence aux objectifs qu'elles poursuivent (Delion 1981).

La deuxième partie présente la méthode des comptes de surplus ainsi que les résultats. La troisième et dernière partie clôture le travail par une conclusion suivie des perspectives d'avenir des entreprises publiques ferroviaires africaines face à la mondialisation de l'économie.

2 Notamment dans le domaine de l'emploi et de la formation intérieure brute du capital fixe. 


\section{La performance des entreprises publiques africaines de chemin de fer}

\subsection{La problématique de l'évaluation}

Il est incontestablement établi que les entreprises publiques africaines de chemin de fer ont des résultats financiers désastreux (Nellis 1988, Banque Mondiale 1994). Toutefois, on doit se garder de tirer des conclusions hâtives de ces résultats en s'appuyant uniquement sur le critère financier. En effet, l'évaluation de la performance de ces entreprises fait l'objet de vives polémiques. C'est essentiellement la controverse sur la rentabilité financière qui se trouve au cœur des débats. Bien que la recherche de la rentabilité doive être encouragée, il reste néanmoins vrai que la notion de perte et profit à elle seule constitue un piètre critère pour mesurer la performance de ces entreprises. Doivent donc être pris en considération la capacité d'incitation à la modernisation, le maintien de l'indépendance des secteurs clés pour le développement national et l'effet d'entraînement sur l'économie.

Il ressort de ce qui précède que les pouvoirs publics africains assignent aux entreprises publiques de chemin de fer l'objectif de profit d'une part et celui d'intérêt général d'autre part.

On notera pourtant que la poursuite de ces objectifs contradictoires est d'autant plus difficile que les Etats connaissent des difficultés budgétaires énormes. Les contraintes de l'environnement public sont, de ce point de vue, fort lourdes. Parmi elles, on relèvera l'instabilité des politiques poursuivies, les très longs circuits décisionnels, la rigidité de l'environnement légal et réglementaire, la multiplicité des procédures et des contrôles, la réglementation des tarifs, etc. Comme ces missions ne sont pas complémentaires mais plutôt conflictuelles, il importe que les pouvoirs publics se fixent préalablement des priorités (Obermann 1999).

En Afrique noire, les voies de communication sont quasi inexistantes. Le déplacement sur de longues distances constitue un luxe pour la majorité de la population, compte tenu de son faible niveau de vie. En effet, plus de $60 \%$ des populations africaines vivent dans des campagnes et ont des revenus très faibles tirés de leurs activités agricoles. Dans la mesure où les transports seraient meilleurs (en qualité et en prix), les termes d'échange des paysans africains ou de tout producteur de l'intérieur du pays s'amélioreraient. De plus, le transport, s'il est offert à un tarif qui tient compte du niveau de vie de la majorité de la population, permet d'accroître les contacts des villageois avec les citadins. Grâce à la mobilité, le transport offre de 
nouveaux modèles de comportement et facilite le déplacement à moindre coût des populations pauvres des banlieues. Grâce à cette mobilité, l'esprit économique et monétaire va se développer et va transformer la topographie des milieux ruraux en bourgs, en faisant reculer la pauvreté. Tous ces éléments plaident pour qu'un service de transport minimum soit garanti dans toutes les régions.

Les pouvoirs publics sont les seuls capables de fournir ces prestations et de pratiquer des prix susceptibles de satisfaire tous les citoyens et de desservir des régions défavorisées. Il est vrai que la poursuite de telles missions implique un coût élevé par usager. C'est à ce niveau que les gouvernants doivent planifier leurs besoins en fonction des ressources disponibles et définir les priorités.

\subsection{Les raisons d'utilisation d'autres critères}

Vu ce qui précède, la prise en compte d'autres critères susceptibles de donner une indication sur la satisfaction des objectifs autres que financier s'avère indispensable (Bridier et Michaïlof 1987).

Le chemin de fer africain constitue un véritable instrument de répartition des ressources productives des pays africains. Il assure la compétitivité des produits africains sur les marchés extérieurs. Il permet d'élargir la gamme des produits exportables et agit sur l'orientation des échanges (Hickling Corporation 1990). Indirectement, son action contribue aussi à la transformation des structures économiques, sociales et politiques existantes. C'est comme cela qu'il exerce des effets d'entraînement sur plusieurs secteurs de l'économie (Button 1993, Israel 1993).

Il est sans conteste que le trafic de marchandises par chemin de fer se trouve étroitement lié aux productions agricoles et minières d'exportation, principales sources de revenu en devises des pays africains (Pourtier 1990). Eu égard à la nature de ces matières premières (produits agricoles en vrac, matériaux de construction, produits forestiers, engrais, produits miniers, etc.), aux distances de transport (en moyenne $650 \mathrm{~km}$ ) et au petit nombre de points entre l'origine et la destination de trafic, le chemin de fer africain répond ainsi d'abord et avant tout à cet objectif de développement économique.

Le transport de minerais de toutes sortes constitue l'espoir d'un grand développement pour la plupart des pays africains. L'accroissement de la production entraîne l'augmentation du volume de trafic, tout comme l'amélioration des conditions de transport a des effets positifs sur la production minière. Le chemin de fer africain offre 
pour le bois, les matériaux de construction et les hydrocarbures, les mêmes avantages que pour les minerais : il transporte à bon marché ces produits lourds et encombrants.

Goffaux indiquait en 1986 (p. 151), . . qu'un vrai développement en Afrique, une économie d'échange, peut être comparée à un organisme vivant dont les infrastructures constituent en quelque sorte le système circulatoire qui irrigue les membres. Toute obstruction, tout enclavement ou isolement entraîne rapidement l'asphyxie économique. Une mauvaise récolte devient alors synonyme de famine tandis qu'une récolte particulièrement abondante verra les surplus pourrir sur place, vu l'impossibilité de les évacuer. L'insuffisance et surtout le mauvais entretien des voies de communications ont pour conséquence que les commerçants ne s'y aventurent plus avec leurs véhicules, ou alors le taux accru d'amortissement de ceux-ci grève lourdement les prix. Dans ces conditions, même une augmentation de la demande urbaine en produits vivriers ne stimulera guère la production. Ce facteur constitue souvent un frein décisif au développement économique de vastes régions'.

Dans le même ordre d'idées, Boiteux (1994) met en évidence le rôle économique des infrastructures par leur contribution au développement économique. Cette contribution se concrétise en premier lieu par l'induction de trafics qu'elles génèrent.

La structure de production des sociétés ferroviaires africaines confirme cette mission première du rail. Le trafic des marchandises représente près de $70 \%$ du total de la production et le trafic de voyageurs environ $30 \%$ (Tableau 1). Une autre caractéristique importante qui apparaît dans la cinquième et la sixième colonnes du tableau 1 est le long parcours de bout en bout effectué par les marchandises et les voyageurs.

L'apport de chemin de fer au développement est ressenti aussi par le désenclavement des régions et par l'amélioration de la qualité de vie. En effet, l'augmentation du taux de desserte de la population apporte une contribution évidente et directe au recul de la souffrance du portage et accroît ainsi la productivité des ruraux.

Une autre mission, d'ordre macro-économique, poursuivie par ces compagnies, est la lutte contre le chômage. C'est ainsi que ces sociétés publiques ont des effectifs qualifiés de pléthoriques par la Banque Mondiale (1994).

Toutes ces raisons poussent à croire que l'évaluation de ces entreprises doit objectivement tenir compte, outre des indicateurs financiers, des indicateurs économiques et sociaux (Rees 1976). 


\section{Tableau 1 - Caractéristiques de la production}

Valeurs moyennessur les périodes

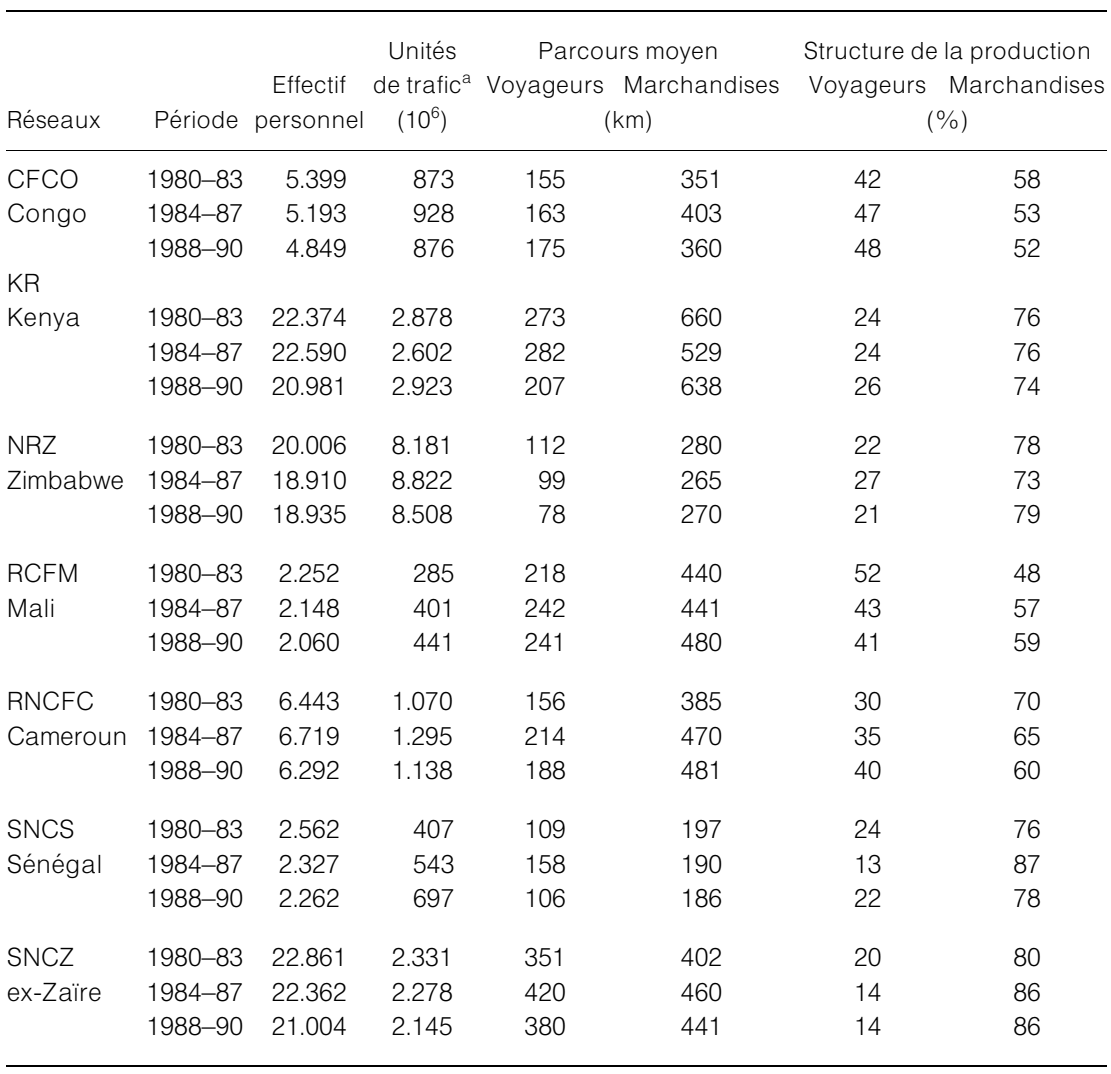

Source: Mbangala 1997.

aL'unité de trafic est la somme des voyageurs-kilomètres (VK) et des tonnes-kilomètres (TK) transportées.

\section{$2 \quad$ La mesure de performance}

\subsection{Le critère de performance}

Les objectifs d'intérêt général des entreprises publiques permettent la redistribution des revenus entre la population ainsi que le développement économique et social. Leurs activités provoquent ainsi des effets d'entraînement dans des secteurs en amont et en aval (Little et Mirrlees 1968). Le critère de performance économique peut bien rendre compte de tels objectifs car l'idée de 'performance économique' comme critère d'évaluation consiste justement à vérifier CCIRIEC 2001 
le degré de réalisation des objectifs de développement économique et social (Hirshihorn et Kaell 1988). Lorsqu'une entreprise poursuit de tels objectifs, il est sous-entendu que sa performance ne peut s'apprécier sur la seule base de l'efficacité relative à l'activité productive. Cette dernière n'est qu'un critère d'appréciation parmi tant d'autres. Une performance technique ou financière qui aboutirait à aggraver la disparité des revenus n'est guère justifiée du point de vue de l'intérêt général. Inversement une activité moins performante (techniquement ou financièrement), qui a pour but de désenclaver une région pauvre et de lui donner une grande chance de participer à la croissance générale du pays, peut être justifiée dès lors que ses chances de réussite sont nombreuses.

\subsection{Le choix d'une mesure de performance}

La mesure de performance basée sur le critère de rentabilité économique n'est pas toujours facile à mettre en cuvre dans la pratique. L'évaluation est rendue difficile d'abord par la nécessité d'une recherche dans les comptes des agents économiques. Ceux-ci ne sont pas nécessairement ouverts à l'investigation, ou n'existent même pas au moment de réaliser cette investigation, et obligent donc à des approximations (Bergmann et Boussard 1994). Problème plus délicat encore, l'existence à côté d'éléments 'marchands' (c'est-à-dire objets de transactions sur le marché), d'éléments 'non marchands' qui doivent alors faire l'objet d'évaluations parfois arbitraires. Même si l'on peut réduire l'incertitude par l'observation des comportements ou par des statistiques et sondages, il reste une part de subjectivité quand l'évaluation concerne la sensation de confort ou d'inconfort, la sécurité et le prix de la douleur ou de la vie.

Plusieurs méthodes ont été développées pour évaluer un projet (ex ante ou ex post) en tenant compte de son impact sur l'économie nationale (Bridier et Michaïlof 1987). Malheureusement la plupart de ces méthodes présentent beaucoup d'inconvénients. Les outils qu'elles proposent sont d'un emploi délicat et exigent des informations statistiques qui sont rarement disponibles dans les pays en voie de développement. Les fondements théoriques de certaines de ces méthodes sont fragiles et contestables. On ajoutera aussi le fait que les décideurs n'ont guère confiance dans des techniques qu'ils connaissent peu ou mal.

Compte tenu de toutes ces difficultés, la méthode des comptes de surplus (CERC 1980) présente quelques avantages. Premièrement, la méthode s'appuie sur les comptes de résultats qui sont moins 
difficilement accessibles. Deuxièmement, elle fournit une vision globale de l'entreprise en tant qu'unité de création et de distribution de richesses. Elle montre d'une part comment l'entreprise utilise les facteurs de production pour maximiser la production. Elle explique d'autre part comment s'organise l'activité de répartition entre tous les fournisseurs des facteurs de production et comment cette production évolue dans le temps.

Elle fait ainsi apparaître la part qui revient à chacun dans l'amélioration réalisée en commun, et facilite le partage des fruits de la croissance entre les divers partenaires sociaux.

Si on arrive à chiffrer et à interpréter les causes des résultats, cette méthode permet de préparer rationnellement de nouveaux progrès et de comprendre la manière dont l'entreprise est gérée par les managers (Lauzel et Teller 1997). ${ }^{3}$

Cependant, la difficulté de la méthode réside dans sa mise en œuvre. La désagrégation des différents postes de comptes de résultats repose sur des hypothèses de prix et de quantités (Geels 1988).

\subsection{La présentation de la méthode}

L'élaboration des comptes de surplus se fait en deux étapes. Dans un premier temps, on calcule le surplus de productivité globale des facteurs. Dans un second temps, on détermine la répartition des avantages et désavantages.

Le surplus de productivité globale des facteurs (SPGF), noté $\sigma$, tient compte des variations des quantités. Il est mesuré par la différence entre la variation des quantités produites et celle des quantités consommées (facteurs de production) d'une année à l'autre. Ces quantités sont évaluées au prix de la première année ou au prix constant.

$$
\sigma=\sum_{i=1}^{I} p_{i} \Delta q_{i}-\sum_{j=1}^{J} d_{j} \Delta k_{j},
$$

avec,

$p_{i}: \quad$ prix des produits $(i=1, \ldots, I)$ correspondant à la première des deux années;

3 Ces auteurs montrent que l'application de la méthode dans le cas d'Electricité de France (EDF), a donné des résultats satisfaisants, p. 215. 
$\Delta q_{i}$ : variation de la quantité produite entre deux années considérées $\left(t\right.$ et $\left.t_{+1}\right)$

$d_{j}$ : prix des facteurs $(j=1, \ldots, J)$ de production correspondant à la première des deux années;

$\Delta k_{j}$ : variation de la quantité du facteur utilisé entre deux années considérées $\left(t\right.$ et $\left.t_{+1}\right)$.

On parlera de gain ou de perte de productivité globale selon que ce surplus est soit positif, soit négatif.

La détermination des avantages et de leur répartition, notés $\alpha$, tient compte de changements de prix d'une année à l'autre. L'effet global des variations de prix se mesure en prenant pour chaque poste du compte d'exploitation (produits et charges) la variation de prix de l'année $t$ à l'année $t_{+1}$ pondérée par la quantité produite au cours de la seconde année. On raisonne donc à quantités constantes.

Dès lors, le surplus à partager consiste en la somme du surplus dégagé au sein de l'entreprise et du surplus externe, prélevé sur les agents extérieurs d'une année à l'autre. Il sera donc déterminé par l'expression suivante :

$$
\alpha=\sum_{i=1}^{I}\left(-\Delta p_{i}\right)\left(q_{i}+\Delta q_{i}\right)+\sum_{j=1}^{J}\left(\Delta d_{j}\right)\left(k_{j}+\Delta k_{j}\right) .
$$

On appellera 'avantage' les suppléments de valeurs obtenus du fait des variations de prix, par les acheteurs des produits de l'entreprise ou par les agents économiques qui lui procurent ses facteurs de production. Ainsi une hausse des prix d'un article :

- est un avantage pour le fournisseur et un désavantage pour l'entreprise si cet article est un facteur de production pour cette dernière;

- est un avantage pour l'entreprise et un désavantage pour le client si cet article est un produit fini de l'entreprise.

Du fait de l'égalité qui résume le compte d'exploitation (Produits = Charges), le Surplus de Productivité Globale des Facteurs (SPGF) sera égal à la somme des avantages à répartir. En partant, pour la première année, de cette égalité comptable, on a :

$$
\sum_{i=1}^{I} p_{i} q_{i}=\sum_{j=1}^{J} d_{j} k_{j}
$$

ou bien 


$$
\sum_{j=1}^{J} d_{j} k_{j}-\sum_{i=1}^{I} p_{i} q_{i}=0
$$

en $t_{+1}$, suite aux variations de prix et de quantités, on obtient :

$$
-\sum_{i=1}^{I}\left(p_{i}+\Delta p_{i}\right)\left(q_{i}+\Delta q_{i}\right)+\sum_{j=1}^{J}\left(d_{j}+\Delta d_{j}\right)\left(k_{j}+\Delta k_{j}\right)=0
$$

et en développant les deux termes de (4) il s'en suit:

$$
\begin{gathered}
-\sum_{i=1}^{I} p_{i} q_{i}+\sum_{j=1}^{J} d_{j} k_{j}-\sum_{i=1}^{I} p_{i} \Delta q_{i}-\sum_{j=1}^{J} d_{j} \Delta k_{j}+-\sum_{i=1}^{I} \Delta p_{i}\left(q_{i}+\Delta q_{i}\right) \\
+\sum_{j=1}^{J} \Delta d_{j}\left(k_{j}+\Delta k_{j}\right)=0,
\end{gathered}
$$

d'où, en se servant des équations (1), (2) et (3), on peut montrer que : $-\sigma+\alpha=0$, et par conséquence $\sigma=\alpha$.

Cette égalité entre le surplus de productivité globale et la somme des avantages constitue le fondement de la méthode des Comptes de surplus.

La valeur du surplus étant fortement liée à la taille de l'entreprise, nous calculons un taux de surplus de productivité globale en le rapportant à la valeur de production de la première année. Il peut également être obtenu en faisant la différence entre le taux d'accroissement du volume de production et le taux d'accroissement du volume des facteurs de production.

\section{$3 \quad$ Le traitement des données et l'analyse des résultats}

\subsection{Le traitement des données}

La méthode s'applique à un échantillon de sept entreprises publiques africaines de chemin de fer sur une période de onze ans, soit de 1980 à 1990. Il s'agit de la société de Chemin de fer Congo-Océan du Congo (CFCO), de la Kenyan Railways du Kenya (KR), de la Régie de Chemins de fer du Mali (RCFM), de la Régie Nationale des Chemins de fer du Cameroun (RNCFC), de la National Railways of Zimbabwe (NRZ), de la Société Nationale du Chemin de Fer du Sénégal (SNCS) et de la Société Nationale du Chemin de fer de l'ex-Zaïre (SNCZ). 
Sur les comptes de résultats de ces sociétés, nous avons effectué trois modifications. La première modification a trait au reclassement des comptes provenant des différentes normes comptables en vue de l'obtention des données homogènes. Ensuite, ces comptes de résultats ont été convertis en une unité monétaire identique, le dollar américain au taux de change officiel, faute de disposer d'indices de conversion mieux adaptés.

Enfin, nous avons effectué quelques restructurations sur ces comptes pour nous conformer à la réalité des entreprises publiques (Michel et Vandenbussche 1988). Toutes les subventions d'exploitation ont été enlevées du compte de produits et mises en soustraction dans les charges du personnel. Les subventions relatives à la réduction du prix imposé aux entreprises pour certaines catégories d'usagers sont considérées comme une contribution de l'Etat à la réalisation du chiffre d'affaires, et sont incorporées dans le poste 'Autres produits d'exploitation'.

Le regroupement des comptes a permis de subdiviser les charges en 4 postes : le carburant comme principale matière première consommée ${ }^{4}$, les charges du personnel, les autres charges (autres consommations intermédiaires, les dotations aux amortissements et provisions et les charges financières) et le résultat de l'exercice. Les comptes de produits sont regroupés en trois postes : les recettes du trafic marchandises, les recettes du trafic voyageurs et les autres recettes qui englobent les compensations de l'Etat et les autres produits d'exploitations (les recettes hôtelières, les ventes de boissons dans le train, etc.).

La décomposition des valeurs en quantités et en prix ne pose aucun problème pour certains postes. C'est le cas des charges du personnel dont on connaît l'effectif. Il n'en va pas de même pour d'autres postes : carburant, autres charges et dotation aux amortissements. Pour ces postes dont les indicateurs de prix ou de quantités spécifiques font défaut, nous avons utilisé un indicateur de prix ou de quantité exogène, en l'occurrence l'indice de prix à la consommation (Geels 1988). Ces indices sont fournis par les statistiques financières de la Banque Mondiale (International Bank of Reconstruction and Development 1996).

4 Suivant l'entretien que nous avons eu avec les responsables de la SNCS, le poste carburant (énergie) comprend près de $60 \%$ du total des consommations intermédiaires. Ainsi, nous avons pris cela comme hypothèse de travail pour toutes les entreprises dont les comptes de résultats sont fournis par nature et non par destination. 
La décomposition des produits en quantités et prix se fait de la manière suivante : pour les recettes du trafic marchandises, l'indicateur de volume retenu est le nombre de tonnes-kilomètres (TK), pour les recettes du trafic voyageurs, l'indicateur de volume retenu est le nombre de voyageurs-kilomètres (VK) et pour les autres produits d'exploitation, l'indicateur de volume pris en compte est l'unité de trafic $(\mathrm{TK}+\mathrm{VK}){ }^{5}$

\subsection{L’analyse des résultats}

Au Tableau 2.a sont présentés les résultats des estimations en terme de surplus de productivité $(\sigma)$ et au Tableau 2.b leur contrepartie en répartition des avantages $(\alpha)$. Tous les résultats correspondent à des moyennes sur la période analysée.

La variation de la productivité globale révèle que les entreprises connaissent des périodes de gains, de pertes ou de baisses de productivité dans la combinaison des moyens de production. Trois situations se dégagent du Tableau 2.a pour expliquer les gains de productivité globale réalisés par ces compagnies.

Dans la première, les gains sont dus au fait que la production a augmenté plus que l'augmentation des facteurs de production. Cela est dû à l'augmentation du volume des trafics marchandises et voyageurs. C'est le cas à la RCFM (83-86; 86-90) et à la RNCFC (80-83).

La deuxième situation de gains de productivité provient du fait que la variation positive de la production est accompagnée d'une variation négative des facteurs de production. On observe cette situation au CFCO (83-86), à la KR (86-90) et à la SNCS (83-86). Dans ces trois compagnies, comme on peut l'observer dans le Tableau 1, l'effectif du personnel a connu une réduction au cours de ces périodes, en même temps qu' augmentait le volume du trafic.

Une troisième situation correspond au cas où la diminution du volume de production est accompagnée d'une diminution, beaucoup plus importante, du volume des facteurs de production. C'est le cas notamment de la SNCZ (80-83, 83-86, 86-90) qui a connu une diminution sensible des matériels de traction, de matériels remorqués et de l'effectif du personnel (Mbangala et Perelman 1997) plus que

5 L'utilisation de cette mesure est préconisée dans d'autres comparaisons internationales, à titre d'exemple, voir l'étude réalisée par le Bureau of Industry Economics (Australia Government Publishing Service 1995). 
proportionnelle à la diminution du volume des trafics marchandises et voyageurs.

Dans le Tableau 2.a, deux phénomènes peuvent expliquer les pertes de productivité globale des facteurs. Le premier de ces phénomènes est observé quand le volume du transport diminue plus rapidement que l'importance des facteurs utilisés dans la production. Cette situation se rencontre au CFCO (86-90) où le volume des trafics a diminué, en termes relatifs, plus que le personnel ou la consommation d'énergie. Le deuxième phénomène correspond à la situation où la croissance du volume de production s'accompagne d'une croissance beaucoup plus importante des quantités de facteurs utilisés. Cette situation se trouve à la SNCS (86-90) et à la NRZ (80-83).

Les résultats du Tableau 2.a indiquent aussi que la productivité globale des facteurs évolue en dents de scie dans certains réseaux (CFCO, KR, NRZ, SNCS et SNCZ) et baisse continuellement dans d'autres (RCFM, RNCFC).

On doit cependant reconnaître que la perte de productivité est due, dans la plupart des cas, à la diminution des trafics. Cette baisse de production est sous l'influence de nombreux facteurs dont certains échappent totalement au contrôle des sociétés elles-mêmes.

Parmi ces facteurs, nous relevons les variables exogènes suivantes:

- La dépendance du chemin de fer des produits miniers et agricoles qui constituent plus de $70 \%$ de sa production. Le rail n'étant pas maître de sa demande, il se retrouve dans une chaîne qui comprend en amont la production des ressources minières et agricoles et en aval l'importation des produits manufacturés, des hydrocarbures et des pièces de rechange, etc. Bref, le chemin de fer joue le rôle d'intendance pour les industries minières et agricoles, caractéristique particulière des économies africaines désarticulées.

- L'enclavement des pays et des sous-régions. Cela résulte du manque de moyens de communication interconnectés et de l'inexistence des voies routières. Il existe, à l'intérieur même des pays, des zones entières non desservies ni par la voie ferrée, ni par le réseau routier. Une telle carence de moyens de transport handicape lourdement les activités agricoles.

A côté des facteurs externes, plusieurs variables internes peuvent également expliquer la baisse de la production ferroviaire. Nous citerons ici les trois causes les plus visibles, à savoir :

- La vétusté des infrastructures et des équipements. Cette détérioration a contribué largement à la limitation de l'offre de 
Tableau 2 - Comptes des Surplus des sociétés de chemin de fer africain (1980-1990)

a. Surplus de productivité (Moyennes sur les périodes, en \% sur la valeur de la production)

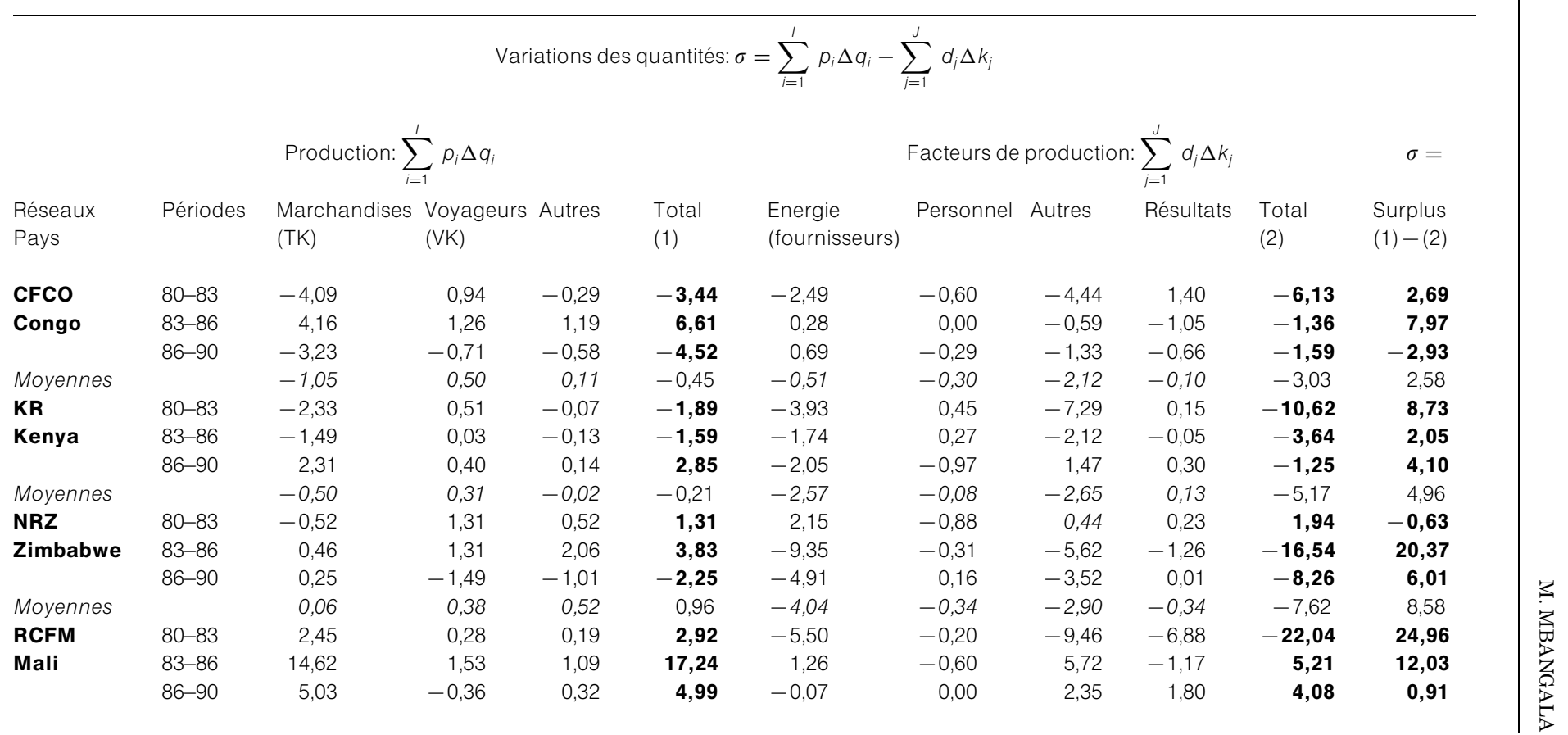




\begin{tabular}{|c|c|c|c|c|c|c|c|c|c|c|c|}
\hline Moyennes & & 7,37 & 0,48 & 0,53 & 8,38 & $-1,44$ & $-0,27$ & $-0,46$ & $-2,08$ & $-4,25$ & 12,63 \\
\hline RNCFC & 80-83 & 7,00 & 3,59 & 3,68 & 14,27 & $-2,70$ & 1,38 & $-1,48$ & 9,94 & 7,14 & 7,13 \\
\hline \multirow[t]{2}{*}{ Cameroun } & $83-86$ & $-1,71$ & 0,18 & 0,01 & $-1,52$ & $-2,44$ & 0,07 & $-4,70$ & 0,91 & $-6,16$ & 4,64 \\
\hline & $86-90$ & $-0,65$ & 0,08 & $-0,40$ & $-0,97$ & $-1,16$ & $-0,96$ & 5,16 & $-5,17$ & $-2,13$ & 1,16 \\
\hline Moyennes & & 1,55 & 1,28 & 1,10 & 3,93 & $-2,10$ & 0,16 & $-0,34$ & 1,89 & $-0,38$ & 4,31 \\
\hline SNCS & 80-83 & 2,26 & $-0,33$ & 0,35 & 2,28 & $-2,72$ & $-0,27$ & $-7,85$ & $-0,85$ & $-11,69$ & 13,97 \\
\hline \multirow[t]{2}{*}{ Sénégal } & 83-86 & 8,12 & $-0,26$ & 3,33 & 11,19 & 2,94 & $-0,74$ & $-7,08$ & 0,16 & $-4,72$ & 15,91 \\
\hline & $86-90$ & 3,40 & 2,38 & 2,79 & 8,57 & 1,37 & 0,81 & 7,89 & 0,25 & 10,32 & $-1,75$ \\
\hline Moyennes & & 4,59 & 0,60 & 2,16 & 7,35 & 0,53 & $-0,07$ & $-2,35$ & $-0,15$ & $-2,03$ & 9,38 \\
\hline SNCZ & 80-83 & 3,38 & 1,27 & 0,79 & 5,44 & $-10,19$ & 0,17 & $-12,15$ & $-0,25$ & $-22,42$ & 27,86 \\
\hline \multirow[t]{2}{*}{ ex-Zaïre } & 83-86 & $-0,98$ & $-1,02$ & $-0,34$ & $-2,34$ & $-7,63$ & 0,04 & $-12,90$ & $-0,46$ & $-20,95$ & 18,61 \\
\hline & $86-90$ & $-1,26$ & $-0,67$ & $-0,46$ & $-2,39$ & $-10,75$ & 0,00 & $-21,39$ & 0,15 & $-31,99$ & 29,60 \\
\hline Moyennes & & 0,38 & $-0,14$ & 0,00 & 0,24 & $-9,52$ & 0,07 & $-15,48$ & $-0,19$ & $-25,12$ & 25,36 \\
\hline
\end{tabular}


Tableau 2 - Comptes des Surplus des sociétés de chemin de fer africain (1980-1990)

b. Répartition des avantages (Moyennes sur les périodes, en \% sur la valeur de la production)

\begin{tabular}{|c|c|c|c|c|c|c|c|c|c|c|c|}
\hline \multicolumn{12}{|c|}{ Variations des prix: $\alpha=\sum_{i=1}^{1}\left(-\Delta p_{i}\right)\left(q_{i}+\Delta q_{i}\right)+\sum_{j=1}^{J}\left(\Delta d_{j}\right)\left(k_{j}+\Delta k_{j}\right)$} \\
\hline \multicolumn{6}{|c|}{ Production: $\sum_{i=1}^{l}\left(-\Delta p_{i}\right)\left(q_{i}+\Delta q_{i}\right)$} & \multicolumn{5}{|c|}{ Facteurs de production: $\sum_{j=1}\left(\Delta d_{j}\right)\left(k_{j}+\Delta k_{j}\right)$} & \multirow{2}{*}{$\begin{array}{c}\alpha= \\
\text { Avantage } \\
(1)-(2)\end{array}$} \\
\hline $\begin{array}{l}\text { Réseaux } \\
\text { Pays }\end{array}$ & Périodes & $\begin{array}{l}\text { Marchandises } \\
(\mathrm{TK})\end{array}$ & $\begin{array}{l}\text { Voyageurs } \\
\text { (VK) }\end{array}$ & Autres & $\begin{array}{l}\text { Total } \\
(1)\end{array}$ & $\begin{array}{l}\text { Fournisseurs } \\
\text { (énergie) }\end{array}$ & Personnel & Autres & Résultats & $\begin{array}{l}\text { Total } \\
(2)\end{array}$ & \\
\hline CFCO & $80-83$ & $-5,41$ & $-2,05$ & $-1,06$ & $-4,42$ & 3,81 & 0,12 & 1,41 & 1,77 & 7,11 & 2,69 \\
\hline \multirow[t]{2}{*}{ Congo } & $83-86$ & 0,25 & $-1,32$ & $-0,77$ & $-1,84$ & 2,10 & 4,18 & 1,55 & 1,98 & 9,81 & 7,97 \\
\hline & $86-90$ & $-4,46$ & $-2,83$ & 0,25 & $-7,04$ & 0,47 & 2,81 & 1,07 & $-0,24$ & 4,11 & $-2,93$ \\
\hline Moyennes & & $-3,21$ & $-0,70$ & $-0,53$ & $-4,43$ & 2,13 & 2,37 & 1,34 & 1,17 & 7,01 & 2,58 \\
\hline KR & $80-83$ & $-1,05$ & 0,56 & 0,73 & 0,24 & 5,68 & $-2,49$ & 0,54 & 4,76 & 8,49 & 8,73 \\
\hline \multirow[t]{2}{*}{ Kenya } & $83-86$ & 1,97 & 0,01 & $-0,38$ & 1,60 & 3,37 & $-0,96$ & 0,34 & $-2,30$ & 0,45 & 2,05 \\
\hline & $86-90$ & $-1,84$ & $-0,57$ & $-1,38$ & $-3,79$ & 4,75 & $-0,19$ & 1,27 & 2,06 & 7,89 & 4,10 \\
\hline Moyennes & & $-0,31$ & 0,00 & $-0,34$ & $-0,65$ & 4,60 & $-1,21$ & 0,72 & 1,51 & 5,61 & 4,96 \\
\hline NRZ & $80-83$ & 1,88 & $-0,10$ & $-12,36$ & $-10,58$ & 7,67 & $-1,55$ & 0,81 & 3,02 & 9,95 & $-0,63$ \\
\hline \multirow[t]{2}{*}{ Zimbabwe } & $83-86$ & 3,66 & 2,00 & 8,61 & 14,27 & 6,28 & $-2,58$ & $-0,05$ & 2,45 & 6,10 & 20,37 \\
\hline & $86-90$ & 0,87 & $-1,63$ & 5,66 & 4,90 & 5,26 & $-0,22$ & 0,40 & $-4,33$ & 1,11 & 6,01 \\
\hline Moyennes & & 2,14 & 0,09 & 0,64 & 2,86 & 6,40 & $-1,45$ & 0,39 & 0,38 & 5,72 & 8,58 \\
\hline RCFM & $80-83$ & 5,29 & 4,57 & 7,73 & 17,59 & 4,82 & $-4,84$ & 1,12 & 6,27 & 7,37 & 24,96 \\
\hline \multirow[t]{2}{*}{ Mali } & $83-86$ & 12,45 & $-2,21$ & $-39,09$ & $-28,85$ & 6,47 & 2,92 & 2,29 & 29,20 & 40,88 & 12,03 \\
\hline & $86-90$ & $-4,83$ & $-4,94$ & 4,77 & $-5,00$ & $-0,59$ & 6,85 & 2,51 & $-2,86$ & 5,91 & 0,91 \\
\hline
\end{tabular}




\begin{tabular}{|c|c|c|c|c|c|c|c|c|c|c|c|}
\hline \multicolumn{2}{|l|}{ Moyennes } & 4,30 & $-0,86$ & $-8,86$ & $-5,42$ & 3,57 & 1,64 & 1,97 & 10,87 & 18,05 & 12,63 \\
\hline RNCFC & 80-83 & 2,18 & 0,13 & 11,76 & 14,07 & 4,42 & 4,24 & 2,47 & $-18,07$ & $-6,94$ & 7,13 \\
\hline \multirow[t]{2}{*}{ Cameroun } & $83-86$ & $-2,05$ & 0,51 & $-2,45$ & $-3,99$ & 2,87 & 4,20 & $-0,17$ & 1,73 & 8,63 & 4,64 \\
\hline & $86-90$ & $-2,61$ & $-0,43$ & $-5,79$ & $-8,83$ & 0,79 & 3,52 & 3,86 & 1,82 & 9,99 & 1,16 \\
\hline Moyennes & & $-0,83$ & 0,07 & 1,17 & 0,42 & 2,69 & 3,99 & 2,05 & $-4,84$ & 3,89 & 4,31 \\
\hline SNCS & 80-83 & 9,42 & 0,41 & 3,21 & 13,04 & 5,29 & $-2,51$ & 1,01 & $-2,86$ & 0,93 & 13,97 \\
\hline \multirow[t]{2}{*}{ Sénéga } & 83-86 & $-4,70$ & $-0,98$ & 3,45 & $-2,23$ & 5,70 & 4,11 & $-0,56$ & 8,89 & 18,14 & 15,91 \\
\hline & $86-90$ & $-0,78$ & $-0,57$ & $-2,89$ & $-4,24$ & $-0,62$ & 2,84 & 0,25 & 0,02 & 2,49 & $-1,75$ \\
\hline Moyennes & & 1,31 & $-0,38$ & 1,26 & 2,19 & 3,46 & 1,48 & 0,23 & 2,02 & 7,19 & 9,38 \\
\hline SNCZ & 80-83 & 13,7 & 2,81 & 7,55 & 24,06 & 12,83 & $-8,35$ & 0,69 & $-1,37$ & 3,80 & 27,86 \\
\hline \multirow[t]{2}{*}{ ex-Zaïre } & 83-86 & $-0,14$ & 1,70 & $-2,25$ & $-0,69$ & 12,35 & $-2,19$ & 0,71 & 8,43 & 19,30 & 18,61 \\
\hline & $86-90$ & 6,10 & 2,11 & 0,97 & 9,18 & 23,52 & $-6,83$ & 0,71 & 3,02 & 20,42 & 29,60 \\
\hline Moyennes & & 6,55 & 2,21 & 2,09 & 10,85 & 16,23 & $-5,79$ & 0,70 & 3,36 & 14,51 & 25,36 \\
\hline
\end{tabular}




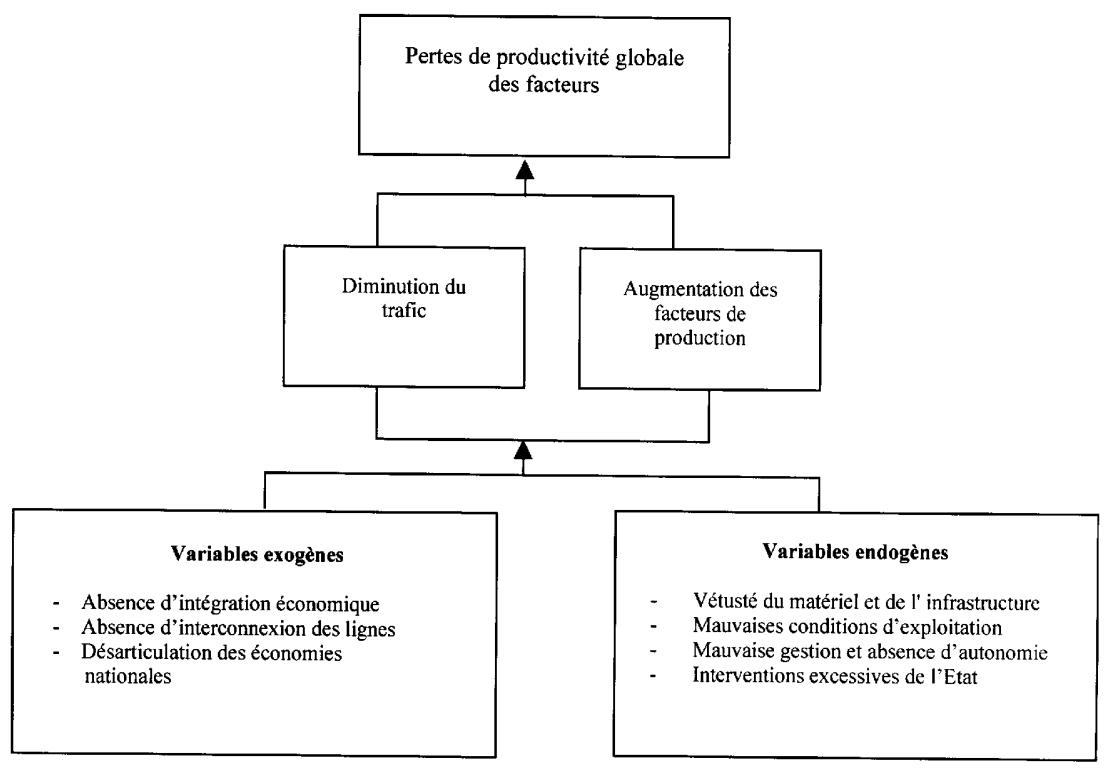

Figure 1. - Origines des pertes de productivité globale des facteurs

transport ferroviaire et a entraîné le développement de la concurrence des autres modes de transport, surtout routier.

- La mauvaise condition d'exploitation. L'absence du respect des délais de livraison des marchandises, de nombreuses pertes et dégâts causés aux marchandises transportées, les retards d'acheminement des marchandises, l'irrégularité des trains de voyageurs, l'entassement des clients dans les voitures, la faible vitesse des trains (50 à $70 \mathrm{~km} /$ heure), etc.

- L'absence d'autonomie de gestion caractérisée par les interventions persistantes des gouvernements sur tous les aspects de la gestion courante. En outre, ces sociétés sont largement sollicitées pour la réalisation des objectifs de la politique économique sans que les moyens nécessaires leur soient fournis (peu de dotation en capital, pressions sur les tarifs, peu ou pas de subvention en équipement, etc.). En même temps, les mêmes dirigeants politiques font souvent passer leur opportunisme et leur individualisme avant la vie des organisations en s'octroyant des dotations dont le contour est illimité, faisant régner de la sorte une oligarchie financière et décapitant dès lors la vie des organisations (Merlin 1996).

Tous les problèmes évoqués sont résumés dans la Figure 1. 
En dépit de pertes ou de baisses de productivité enregistrées, les fournisseurs d'énergie semblent être les premiers bénéficiaires des avantages créés par ces compagnies sur toutes les périodes, à l'exception de la NRZ (80-83), de la RCFM (86-90) et de la SNCS (8690) (Tableau 2.b). Ces avantages s'expliquent par le fait que la diminution de volume d'énergie consommée par les entreprises a été compensée par une augmentation, en termes réels, du prix de cette consommation intermédiaire.

Les travailleurs de la KR, de la NRZ et de la SNCZ ne sont pas des bénéficiaires des avantages créés par leurs sociétés respectives car la baisse des effectifs n'a pas été compensée par une augmentation de salaires. Le tableau 2.b indique par contre que le CFCO, la RCFM, la RNCFC et la SNCS ont connu une croissance réelle de leur salaire (moyenne des sous-périodes).

Pour le trafic marchandises, on constate que dans certains cas la diminution de volume du trafic, observée dans le Tableau 2.a, est accompagnée d'une augmentation des tarifs en termes réels. Sauf à la NRZ, à la RCFM et à la SNCZ où le trafic marchandises bénéficie des avantages (moyenne des sous-périodes)

En ce qui concerne le trafic voyageurs, les tarifs augmentent en termes réels dans la plupart des réseaux, à l'exception de la KR et de la RNCFC (pour les deux premières sous-périodes) dont les usagers voyageurs bénéficient de (très faibles) avantages. Seule la SNCZ connaît une situation où les voyageurs bénéficient d'un avantage, sur toutes les sous-périodes, en dépit d'une diminution du volume du trafic (Tableau 2.a).

\section{Conclusion}

Cet article montre que l'évaluation de la performance des entreprises publiques ne peut se faire de manière uniforme en se basant sur le seul critère financier. Elle nécessite des mesures d'appréciation pertinentes et suffisamment élaborées qui tiennent compte de tous les objectifs. Dans cet article, nous avons évalué la performance des chemins de fer africains au regard de l'objectif de rentabilité économique par l'utilisation de la méthode des comptes de surplus.

Les résultats mettent en évidence le rôle de production et de distribution des sociétés ferroviaires africaines respectivement sous la forme de variation de productivité globale des facteurs et de répartition des avantages entre les partenaires de ces entreprises. 
Cependant, ces résultats doivent être relativisés en raison des hypothèses retenues pour dissocier les valeurs en quantité et en prix.

En ce qui concerne les résultats proprement dits, les sociétés connaissent des périodes de gains et de pertes (baisses) de productivité globale de leurs facteurs. Globalement, nous pouvons retenir que ces pertes (baisses) de productivité sont dues principalement au fait que la diminution de la production des sociétés ne s'accompagne pas d'une diminution proportionnelle des facteurs de production.

Par ailleurs, les résultats obtenus indiquent que même si les sociétés sont confrontées à des pertes de productivité globale des facteurs, les avantages existent toujours pour un ou plusieurs partenaires de ces sociétés à cause de l'égalité comptable entre la variation de productivité globale des facteurs et la somme des avantages (positifs ou négatifs). C'est principalement les fournisseurs d'énergie et dans une moindre mesure les travailleurs qui sont les bénéficiaires des richesses créées par les compagnies ferroviaires africaines.

En ce qui concerne la stratégie à adopter pour accroître la production et maîtriser la gestion des facteurs de production, nous préconisons que les Etats africains redéfinissent la politique des transports aux niveaux national et sous-régional et améliorent leurs relations de gestion avec les managers.

En effet, la configuration actuelle des infrastructures de transports ${ }^{6}$ en Afrique noire pose de sérieux problèmes à la fois de coordination des moyens de transports et de développement économique et social. Les routes principales sont parallèles au chemin de fer et lui font une concurrence ardue en présence d'une demande solvable limitée, alors que les routes secondaires dites de dessertes agricoles, qui sont censées lui être complémentaires pour accroître la demande $^{7}$, font défaut.

Le but de la coordination est d'abord d'éviter le double emploi des infrastructures existantes, mais aussi des suréquipements en présence de projets qui répondent à des besoins similaires en un même endroit. En effet, le suréquipement sous cette forme provoque, dans la phase de

\footnotetext{
$6 \quad$ Nous ne traitons ici que la coordination entre le rail et la route.

7 Nous sommes convaincus que l'insuffisance des infrastructures de transports et leurs mauvaises conditions d'exploitation rendent la demande mal connue.
} 
l'exploitation des infrastructures, une concurrence ruineuse et difficile à maîtriser. Donc finalement, un gaspillage pour l'économie.

Nous nous rallions aux résolutions du troisième séminaire repères (Rail International 1994: 27) qui confirment que 'Les chemins de fer africains devraient, tant dans l'intérêt de l'économie nationale que dans leur intérêt commercial propre, se spécialiser dans les domaines d'activité où ils peuvent offrir des services adaptés aux besoins de la clientèle dans des conditions de coûts et de prix compétitifs'. Cela nous amène à proposer une politique de spécialisation de chemin de fer dans des produits pondéreux et dans le trafic de voyageurs à longue distance et de banlieue.

L'amélioration de la production passe aussi par l'adoption d'une approche qui relie plus étroitement la demande à l'exploitation. Doit être prise en compte ici la fiabilité de service. Pour les utilisateurs, cette fiabilité veut dire que le service doit être dans ce cas disponible en quantité, en qualité et au moment voulu car les utilisateurs décident indirectement de la production par le biais du niveau de la qualité de service qui leur est offert.

L'amélioration de la gestion suppose l'amélioration des relations entre les pouvoirs publics et leurs managers. Dans le cadre de cette étude, la réflexion est axée sur la déréglementation de la gestion du rail basée sur l'établissement d'une concession d'exploitation entre l'Etat et un manager privé.

Le principal objectif de la mise en concession de l'activité de l'entreprise publique est d'accroître son efficacité en confiant la gestion à un opérateur privé. Ce dernier, soumis aux disciplines de gestion de type résolument commercial, va orienter l'activité vers la satisfaction des besoins de marché. Cette formule permet également de clarifier les relations entre l'Etat et l'opérateur privé. Il s'agit de remplacer la relation verticale ${ }^{8}$ par une relation horizontale. Celle-ci, basée sur la signature d'un contrat entre deux partenaires autonomes - pouvoirs publics et opérateur privé - interdit l'ingérence de l'Etat dans la gestion de l'exploitation.

Cette mise en concession serait, enfin, l'occasion d'assainir la gestion des activités du secteur public en Afrique noire (Nellis 1989). Ce schéma pourra, dans une certaine mesure, résoudre les problèmes

8 Relation d'ingérence, qui fait perdre aux managers publics leur autonomie de gestion. Cette relation est, dans une proportion importante, à l'origine de la mauvaise gestion des entreprises publiques à cause notamment du clientélisme très ancré dans le secteur public africain. 
de financement et de la pléthore du personnel que l'on rencontre dans ces entreprises.

Pour que cette déréglementation réussisse, il importe qu'une étude approfondie soit menée sur sa mise en application par l'installation d'un cadre réglementaire et institutionnel approprié, à l'instar de ce qui se fait dans l’Union Européenne (Debande et Monami 1999).

\section{REFERENCES}

BANQUE MONDIALE, 1994, Rapport sur l'infrastructure des pays en développement, Washington.

BERGMANN H. et BOUSSARD J. M., 1994, Guide de l'évaluation économique des projets d'irrigation, OCDE, version révisée.

BOITEUX M. (Présidé par), 1994, Transports : pour un meilleur choix des investissements, La Documentation Française, édité par Le Commissariat Général du Plan, Paris.

BRIDIER M. et MICHAILOF S., 1987, Guide pratique d'analyse de projets. Evaluation et choix des projets d'investissements, 4ème édition, Economica, Paris, 1987.

BUREAU OF INDUSTRY ECONOMICS, 1995, Rail Freight, International Benchmarking, Report 95/22, Australia Government Publishing Service.

BUTTON K. J., 1993, Transport Economics, 2nd ed., University Press, Cambridge.

CERC, 1980, 'Productivité globale et comptes de surplus', Document $d u$ CERC (Centre d'Etudes des Revenus et des Coûts), no. 55/56.

DEBANDE O. et MONAMI, 1998, 'Le réglementation et l'organisation du transport des personnes par chemins de fer dans l'Union européenne : entre diversité et convergence', Annals of Public and Cooperative Economics, vol. 69, 3, pp. 347-371.

DELION A. G., 1981,'Les entreprises publiques et le concept d'efficacité', Revue française d'administration publique, no. 20, pp. 13-30.

FINIFTER R. et TREVOUX M., 1992,'Les spécificités des privatisations en Afrique', Marchés Tropicaux du 13 mars.

GEELS Th., 1988,'Analyse de la productivité globale et de la répartition des avantages dans les six sociétés belges de transports urbains par la méthode des comptes de surplus', dans Thiry B. et Tulkens H. (éditeurs), La performance économique des sociétés belges de transports urbains, CIRIEC Section belge, pp. 196-252. 
GOFFAUX J., 1986, Problèmes de développement, CRP, Kinshasa.

HICKLING CORPORATION, 1990, 'Infrastructure in the Developing Countries. A Review of Current Condition and Trends', Rapport établi pour la Banque Mondiale, Département de l'infrastructure et du développement urbain, Washington.

HIRSHIHORN R. and KAELL A., 1988, 'A Framework for Evaluating Public Corporations', Annales de l'économie publique, sociale et coopérative, 2, pp. 141-155.

INTERNATIONAL BANK FOR RECONSTRUCTION AND DEVELOPMENT - The World Bank, Socio-economic Time-series Access and Retrieval System, June 1996.

ISRAEL A., 1993, 'La gestion de l'infrastructure. Les enjeux des années 90', Document de travail de la Banque Mondiale, Washington.

KEMPE RONALD HOPE SR, 1999, 'Human resource management in Botswana: approaches to enhancing productivity in the public sector', International Journal of Human Resource Management, vol. 10, no. 1, February, pp. 108-21.

LAUZEL P. et TELLER R., 1997, Contrôle de gestion et budgets, Sirey, $8^{\text {ème }}$ édition.

LITTLE I. M. D. and MIRRLEES J. A., 1968, Project appraisal and planning for developing countries, Heinemann Educational Books, London.

MBANGALA M., 1997, Objectifs multiples et mesures de performance des entreprises publiques dans les pays en développement. Cas des chemins de fer de l'Afrique noire, Thèse de doctorat, Université de Liège.

MBANGALA M. et PERELMAN S., 1997, 'L'efficacité technique des chemins de fer en Afrique subsaharienne: une comparaison internationale par la méthode de DEA', Revue d'Economie de Développement, 3, pp. 91-115.

MERLIN P., 1996, Espoir pour l'Afrique noire, Présence Africaine.

MICHEL P. et VANDENBUSSCHE P., 1988, 'Analyse financière des six sociétés belges de transports urbains' dans Thiry B. et Tulkens H. (éditeurs), 'La performance économique des sociétés belges de transports urbains', CIRIEC Section belge, pp. 271-313.

MISHRA R. K. and RAVISHANKAR S. (editors), 1986, Public Enterprises in the World, Himalaya Publishing House, New Delhi.

NATIONS UNIES, 1986, Rôle du secteur public dans la mobilisation des ressources financières dans les pays en développement, New York, TCD/ SEM. 
NELLIS J. R., 1988, “Les entreprises publiques dans l’Afrique au Sud du Sahara", Document de travail de la Banque Mondiale, Washington, D.C.

NELLIS J., 1989, 'Contract Plans and Public Enterprise Performance', Document de synthèse de la Banque Mondiale, Washington.

NKANA R. G., 1994, 'L'union africaine des chemins de fer. Stratégie pour les chemins de fer', Rail International no des Juin-Juillet, 25 ème année, sur le Troisième séminaire REPERES du 10 au 14 janvier à New Delhi, pp. 25-37.

OBERMANN G., 1999,'Sector-specific Regulation from a Public Choice Perspective with Regard to the Supply of Services of Public Interest', Annals of Public and Cooperative Economics, vol. 70, no. 2.

POURTIER R., 1990, 'Transportation and Development in Zaïre', Afrique Contemporaine, no. 153 (1st Quarter).

REES R., 1976, Public Enterprise Economics, Weidenfeld and Nicholson, London.

\section{Evaluation of Economic Efficiency of African Public Enterprises through the Total Factor Productivity Method}

This paper focuses on the assessment of the economic performance of seven African railway enterprises using a Total Factor Productivity index decomposition. This method shows the role of these public enterprises in the production and distribution of added value. The analysis allows to identify the, positive or negative, contribution of each production factor to productivity growth. At the same time, it shows how the firm's partners do, or do not benefit from the added value created by these companies.

\section{Bewertung der ökonomischen Effizienz afrikanischer öffentlicher Unternehmen mit Hilfe der Total Factor Productivity-Methode}

Dieser Beitrag befasst sich mit der Bewertung der ökonomischen Performance von sieben afrikanischen Eisenbahnunternehmen, indem die Total Factor Productivity-Methode angewandt wird. Mit dieser Methode lässt sich zeigen, wie die Eisenbahnen Wert schaffen und verteilen. Die Analyse beleuchtet den Beitrag jedes Faktors bei der Erzielung von Gewinn oder dem Verlust von Produktivität. Gleichzeitig zeigt sie die verschiedenen Partner auf, die von den Vorteilen, welche von diesen Unternehmen geschaffen werden, profitieren. 
La evaluación de los resultados económicos de las empresas públicas africanas por el método de las cuentas de excedentes

Este artículo utiliza el método de las cuentas de excedentes para evaluar el resultado económico de siete empresas ferroviarias africanas. Este método muestra el papel de estas empresas públicas en la creación y distribución de riqueza. El análisis pone en evidencia la contribución de cada factor para la obtención de ganancias o generación de pérdidas de productividad. Al mismo tiempo, indica los diferentes miembros que se benefician de las ventajas creadas por las compañias de ferrocarril. 